\title{
Formação online para o desenvolvimento do Pensamento Computacional em professores de Matemática
}

\author{
Thiago Barcelos ${ }^{1}$, Rodrigo Bortoletto ${ }^{1}$, Mary Grace Andrioli ${ }^{2}$ \\ ${ }^{1}$ Laboratório de Tecnologias para Interação \\ Instituto Federal de São Paulo - Câmpus Guarulhos \\ Av. Salgado Filho, 3501, Guarulhos, SP, Brasil - CEP 07115-000 \\ ${ }^{2}$ Instituto Federal de São Paulo - Câmpus Boituva \\ Av. Zélia de Lima Rosa, 100, Boituva, SP, Brasil - CEP 18550-000 \\ \{tsbarcelos, bortoletto, maryg\}@ifsp.edu.br
}

\begin{abstract}
As far as the discussion about incorporating Computational Thinking into basic educational levels is concerned, one of the greatest challenges nowadays is training teachers adequately to develop related activities. In this article we present an online course aimed at pre-service and in-service teacher training, based on building digital games, to enable them to develop activities that present Math topics together with the development of Computational Thinking skills. Preliminary results indicate that participants acquired advanced skills related to some topics of Computational Thinking. Besides that, it was possible to identify that participants had a higher interest on activities directly related to building digital games.
\end{abstract}

Resumo. No contexto das discussões sobre a incorporação do Pensamento Computacional na educação básica um dos principais desafios atuais é a adequada formação de professores para desenvolver atividades relacionadas. Neste artigo apresentamos um curso para formação inicial e continuada de professores de Matemática, baseado na construção de jogos digitais e oferecido em uma plataforma online, visando capacitá-los a criar atividades que envolvam tópicos matemáticos juntamente com o desenvolvimento de competências do Pensamento Computacional. Os resultados preliminares indicam que os participantes atingiram um nivel avançado de competência em alguns tópicos do Pensamento Computacional e tiveram maior interesse por atividades diretamente relacionadas com a construção de jogos.

\section{Introdução}

Desde a proposta inicial de Jeanette Wing de classificar como Pensamento Computacional (PC) um conjunto de competências e habilidades do cientista da computação que poderiam ser desenvolvidas em estudantes da educação básica, uma série de iniciativas vem sendo desenvolvidas para efetivar a difusão de tais competências e habilidades junto a esse público. Dentre elas, podemos destacar a incorporação de diretrizes curriculares para o desenvolvimento do PC ao currículo de referência para o ensino de Computação na educação básica norte-americana (CSTA, 2011) e a inclusão pelo Departamento de Educação do Reino Unido de aspectos do PC 
V Congresso Brasileiro de Informática na Educação (CBIE 2016)

Anais dos Workshops do V Congresso Brasileiro de Informática na Educação (CBIE 2016)

relacionadas à programação de computadores na educação básica para turmas de alunos a partir de cinco anos (CELLAN-JONES, 2014).

Por outro lado, possíveis relações entre o PC e conteúdos tradicionalmente presentes no currículo escolar têm sido identificadas. Há relatos na literatura de estudos que associam o desenvolvimento do PC a tópicos de biologia (QIN, 2009), de redação (HOWLAND; GOOD; BOULAY, 2015) e de matemática (KE, 2014). Em geral, tais estudos argumentam que o desenvolvimento do PC pode ser um meio de suporte ao aprendizado de conteúdos escolares "tradicionais", justificando dessa forma sua incorporação às atividades escolares.

No entanto, uma limitação não trivial à difusão do Pensamento Computacional é a capacitação de professores para a criação e aplicação de atividades de desenvolvimento do PC (BARCELOS et al., 2015; CURZON et al., 2014; YADAV et al., 2014). Essa tarefa pode ser mais desafiadora ao se considerar a carência de professores especializados no ensino de Computação na educação básica e a necessidade de articular o PC com o currículo tradicional obrigatório, como mencionado anteriormente.

Frente ao exposto, este artigo apresenta o desenvolvimento e aplicação piloto de um curso online para a formação inicial e continuada de docentes de Matemática que desejem incorporar temas relacionados ao Pensamento Computacional às suas atividades didáticas. $\mathrm{O}$ curso utilizou como tema motivador o desenvolvimento de jogos digitais, explicitando e discutindo a cada atividade proposta os tópicos e conceitos matemáticos presentes. A sequência deste artigo é organizada da seguinte forma: na seção 2 é apresentada a fundamentação teórica para a necessidade de iniciativas de formação docente na área de PC, bem como os pressupostos pedagógicos para a estruturação da formação proposta neste artigo. Na seção 3 a estrutura e atividades do curso online são apresentados. Na seção 4 é descrito um primeiro oferecimento da formação, realizado no segundo semestre de 2015, e uma análise preliminar dos resultados obtidos pelos participantes. Por fim, na seção 5 são apresentadas as conclusões e perspectivas de trabalhos futuros.

\section{Fundamentação teórica}

Segundo Yadav et al. (2014), a formação de professores visando a compreensão e aplicação de conceitos do Pensamento Computacional deve envolver não apenas os professores de Computação mas também os professores de áreas específicas. Os autores utilizaram um quase-experimento ${ }^{1}$ cuja conclusão indicou que professores em formação que participaram de uma oficina de conceitos relacionados ao Pensamento Computacional passaram a conceituá-lo como uma estratégia para resolução de problemas, aplicável a diversas áreas. Outros trabalhos que também visam identificar a mudança de percepção de professores em formação sobre o PC foram relatados por Morreale e Joiner (2011) e Blum e Cortina (2007).

Barcelos et al. (2015) apresentaram uma revisão sistemática da literatura de trabalhos em língua inglesa, na qual concluem a carência de estudos que envolvam a incorporação do Pensamento Computacional na formação de professores de

\footnotetext{
1 Experimento no qual, por razões éticas ou de logística, não é possível atribuir os indivíduos aleatoriamente aos grupos experimentais ou de controle (SHADISH; COOK; CAMPBELL, 2002).
} 
V Congresso Brasileiro de Informática na Educação (CBIE 2016)

Anais dos Workshops do V Congresso Brasileiro de Informática na Educação (CBIE 2016)

Matemática. No contexto brasileiro, Farias, Andrade e Alencar (2015) identificaram, por meio da aplicação de um questionário, que egressos de um curso de Licenciatura em Computação tem pouco conhecimento teórico ou experiência prática em relação ao conceito e desenvolvimento do Pensamento Computacional na educação básica.

Por outro lado, encontram-se evidências da literatura de possíveis aproximações entre as competências e habilidades normalmente associadas à Matemática e aquelas associadas ao Pensamento Computacional. Ke (2014) discute que a utilização do ambiente de programação Scratch para construção de jogos permite que crianças tenham contato com conceitos matemáticos tanto simbólicos quanto concretos, e que a modificação do código de programas demanda um raciocínio abstrato e a interação com o problema por meio de símbolos e expressões matemáticas. Isso vem a complementar o trabalho de Mor e Noss (2008), onde se discute que a linguagem algorítmica pode se constituir como uma "transição suave" entre a imprecisão da linguagem coloquial e o formalismo da linguagem matemática. Comparando as diretrizes curriculares para o ensino de Matemática do Brasil, Estados Unidos e Chile, Barcelos e Silveira (2014) identificaram competências comuns entre as propostas no âmbito do Pensamento Computacional e aquelas definidas como resultado do ensino de Matemática na educação básica.

A utilização da construção de jogos digitais como aspecto motivador de uma formação se justifica pela presença e relevância dos jogos no cotidiano das crianças e adolescentes que hoje frequentam a educação básica. Segundo Peppler e Kafai (2009), os jogos digitais atualmente se constituem como uma "mídia cultural" e que compreender as características e mecanismos dessa mídia faz parte de uma educação crítica. Ainda, deve-se considerar que os jogos também fizeram e fazem parte do cotidiano dos estudantes do ensino superior matriculados em cursos de formação de professores, considerando a faixa etária em que se encontram tais estudantes. Por exemplo, uma pesquisa de mercado realizada em 2012 indica que 51\% dos consumidores de jogos digitais no Brasil tem mais de 19 anos (SEBRAE, 2012). Dessa forma, espera-se que a construção de jogos digitais se constitua como uma temática significativa e motivadora para esse público.

\section{Estrutura do curso online}

O curso foi formatado como uma ação oferecida pela Coordenadoria de Extensão do Câmpus Guarulhos do Instituto Federal de São Paulo (IFSP). As inscrições do curso foram abertas tendo como público-alvo prioritário docentes que atuam na rede pública de ensino com o ensino de Matemática e alunos de Licenciatura em Matemática que tem interesse na aplicação de Tecnologias de Informação e Comunicação (TICs) juntamente com estratégias de ensino inovadoras. Considerando as características do público-alvo, optou-se pelo uso do Ambiente Virtual de Aprendizagem Moodle como plataforma de suporte dos conteúdos a serem apresentados, flexibilizando o local e horário de estudo dos participantes.

O curso foi estruturado em dez módulos, liberados semanalmente no ambiente Moodle. Considerando o prazo para elaboração e entrega do projeto final, a duração total do curso foi de dez semanas. Nos cinco primeiros módulos, os participantes são introduzidos a conceitos fundamentais da programação de computadores, como variáveis, estruturas de seleção e condições lógicas. Foi utilizado o ambiente de 
V Congresso Brasileiro de Informática na Educação (CBIE 2016)

Anais dos Workshops do V Congresso Brasileiro de Informática na Educação (CBIE 2016)

programação Scratch como suporte às atividades de programação. O conteúdo de cada módulo é apresentado na Tabela 1.

Tabela 1. Programação do curso

\begin{tabular}{|l|l|}
\hline Módulo & Conteúdo \\
\hline 1 & $\begin{array}{l}\text { Utilização do ambiente Scratch - ambientação, comandos básicos, uso de } \\
\text { sprites e detecção de colisão entre sprites }\end{array}$ \\
\hline 2 & $\begin{array}{l}\text { Variáveis - conceito, operações, utilização para controle de pontuação. Plano } \\
\text { cartesiano no Scratch, geração de números aleatórios, estrutura condicional }\end{array}$ \\
\hline 3 & $\begin{array}{l}\text { Testando condições e o estado do jogo - operadores lógicos E e OU, estrutura } \\
\text { condicional aninhada }\end{array}$ \\
\hline 4 & Movimentação do personagem - controle por teclas e scroll de tela \\
\hline 5 & Programando um lançamento oblíquo de forma iterativa no Scratch \\
\hline 6 & Abordagens pedagógicas \\
\hline 8 & Design Universal para aprendizagem \\
\hline 9 & $\begin{array}{l}\text { Conectando o Scratch com dispositivos externos: Makey Makey e Kinect - } \\
\text { configuração e possibilidades }\end{array}$ \\
\hline 10 & Design Instrucional - fundamentos e práticas \\
\hline
\end{tabular}

As atividades dos cinco primeiros módulos foram desenvolvidas com base nas diretrizes discutidas em (MUNOZ et al., 2015): todas as atividades se baseiam na construção das mecânicas de jogos digitais; as atividades visam, progressivamente, a construção de um jogo completo (que foi o objeto do projeto final do curso); as atividades introduzem novos conceitos à medida que demandam que os participantes voltem a utilizar conceitos aprendidos nos módulos anteriores; a mecânica dos jogos desenvolvidos traz referências à mecânica de jogos reais e significativos para os estudantes.

Cada atividade foi disponibilizada no ambiente virtual com uma ficha descritiva da atividade e um vídeo, com duração entre 15 e 20 minutos, onde a construção inicial das funcionalidades propostas para o módulo em questão é apresentada. Cada vídeo é sempre dividido em três segmentos. No primeiro, o objetivo da atividade é apresentado. No segundo, denominado "Minuto da Matemática", a relação entre o jogo e algum conceito matemático empregado é formalizada e discutida. No terceiro segmento, denominado "Agora é a sua vez", uma proposta de acréscimo ou melhoria à funcionalidade desenvolvida na aula é deixada para o aprofundamento do estudo dos participantes. Na Figura 1 é apresentado um exemplo do segundo segmento de um vídeo - no módulo 5, onde é proposta a construção da mecânica do jogo Angry Birds, a definição da movimentação do pássaro por meio de um lançamento oblíquo e sua modelagem por meio do cálculo vetorial são apresentados de forma a contextualizar para o participante a relação entre o conteúdo matemático e o código que será desenvolvido posteriormente. 

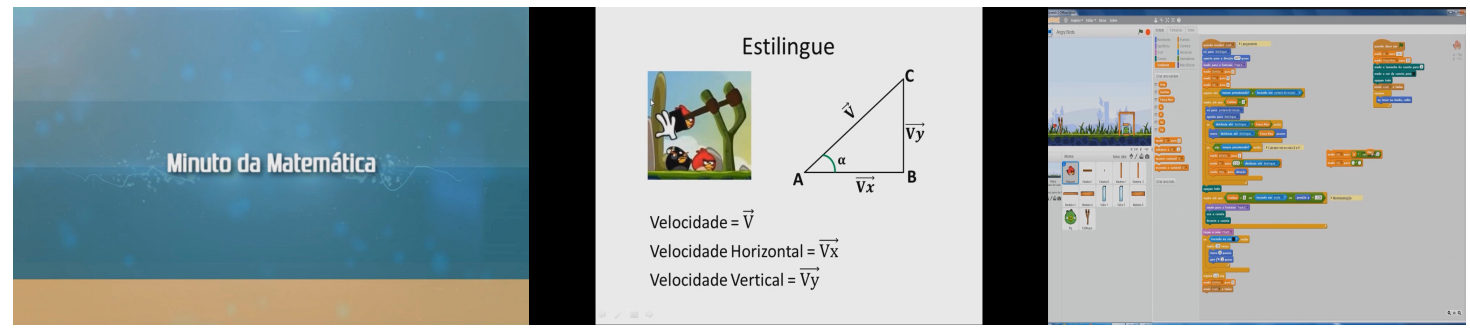

Figura 1. Sequência de apresentação de conteúdo matemático e construção do jogo no material didático em vídeo

Os quatro últimos módulos tiveram como objetivo aprofundar a reflexão dos participantes em relação às atividades a serem desenvolvidas por eles no projeto final do curso e, futuramente, junto a seus alunos. Dessa forma, foi apresentado no módulo 6 um panorama de estratégias pedagógicas para o desenvolvimento de objetos de aprendizagem em geral e no módulo 7 princípios para o Design Universal. No módulo 8 foram apresentados princípios de Design Instrucional para o desenvolvimento de jogos digitais com finalidade educativa, e no módulo 9 foram demonstradas possibilidades de integração do Scratch com os dispositivos Makey Makey e Kinect.

O Makey Makey é um dispositivo de entrada simples desenvolvido com base em Arduíno que possibilita criar uma extensão para o mouse e teclado a partir de objetos do cotidiano. Tanto o Makey Makey quanto o sensor de movimento Kinect da Microsoft podem ser utilizados em aplicações desenvolvidas em Scratch e, por esta razão, foram propostos desafios aos cursistas para a criação de jogos que posteriormente podem utilizar essas possibilidades, mesmo que o participante não tenha acesso aos dispositivos no momento.

Após desenvolver as atividades dos nove módulos os participantes devem desenvolver um jogo de sua própria autoria como projeto final do curso. Essa atividade tem como objetivo a consolidação dos conhecimentos adquiridos ao longo do curso, bem como permitir a contextualização das relações entre a Matemática que faz parte da prática docente do participante e os conceitos do Pensamento Computacional adquiridos. Para tanto, o projeto deve ser desenvolvido com base em três diretrizes: utilizar atores (sprites) em movimento; utilizar três dentre as estruturas de programação aprendidas (variáveis, estruturas condicionais, estruturas de repetição, sincronização via envio de mensagens); o jogo pode ser pensado para ser construído pelos alunos do participante ou para ser jogado por eles. Neste oferecimento do curso a apresentação dos projetos finas foi presencial e teve como aspecto motivador uma premiação para o projeto mais criativo e com melhor jogabilidade.

\section{Resultados preliminares}

O curso teve seu primeiro oferecimento experimental no segundo semestre de 2015. A divulgação das inscrições foi realizada junto a escolas públicas do município de Guarulhos e aos alunos do curso de Licenciatura em Matemática oferecido pelo Câmpus Guarulhos do IFSP. Foram oferecidas 20 vagas para esse primeiro oferecimento. Seguindo regras da instituição, a seleção de participantes para cursos de extensão é feita por sorteio. Além disso, foi feita uma reserva inicial de 50\% das vagas para professores da rede pública, que poderiam ser preenchidas por participantes de outros perfis em caso de não preenchimento em primeira chamada. Ao final do processo de seleção, as vagas 
V Congresso Brasileiro de Informática na Educação (CBIE 2016)

Anais dos Workshops do V Congresso Brasileiro de Informática na Educação (CBIE 2016)

foram preenchidas por 5 professores da rede pública, 14 alunos de cursos de Licenciatura em Matemática ou Pedagogia e 1 aluno de curso superior na área de Informática.

Como um indicativo do envolvimento dos participantes, foram obtidas as estatísticas de acesso aos vídeos, apresentadas na Figura 2. Verifica-se que o acesso aos materiais do curso foi razoavelmente constante, com um pico de acesso no Módulo 1. Verifica-se um acesso maior nos cinco primeiros módulos (média de 33,6 acessos por módulo), os quais envolveram atividades práticas relacionadas aos conceitos de programação. O acesso volta a crescer no segundo vídeo do Módulo 8, também relacionado à construção de um jogo que pode ser integrado ao dispositivo Makey Makey. Uma possível explicação para esse resultado seria um maior interesse dos participantes pelas atividades práticas que envolviam diretamente a construção de jogos.

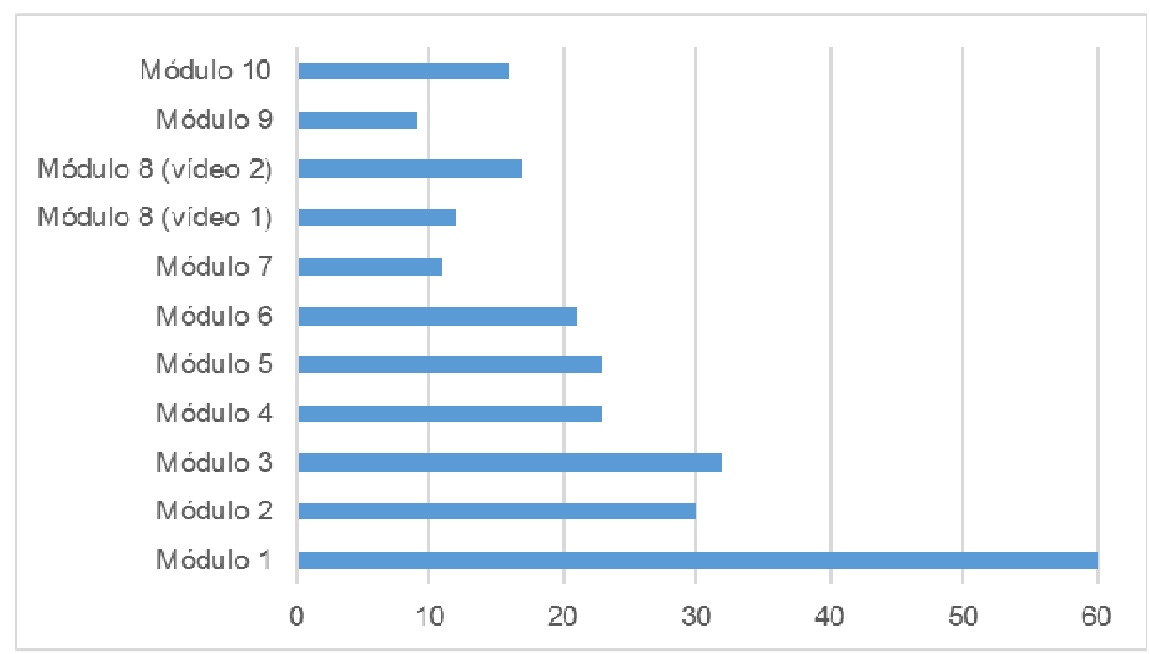

Figura 2. Estatísticas de acesso aos vídeos de cada módulo do curso

No entanto, o acesso aos materiais foi maior do que a entrega de atividades pelos participantes, vinculada às tarefas propostas nos vídeos dos módulos 1 a 5,8 e 10 (projeto final). Cada participante deveria efetuar uma entrega por módulo, e verificou-se uma média de 7,42 entregas por módulo (ou 37,1\% dos participantes inicialmente inscritos), sendo que 5 participantes ( $25 \%$ dos inicialmente inscritos) entregaram o projeto final. Considerando a temática aberta do projeto final, optou-se por realizar uma análise dos artefatos produzidos pelos participantes (BRENNAN; RESNICK, 2012) para evidenciar o desenvolvimento de competências e habilidades do Pensamento Computacional por eles. Para tanto, utilizou-se a rubrica proposta por Moreno-León e Robles (2015), que propõe identificar o uso de conceitos em nível básico, intermediário e avançado em sete categorias. Na Tabela 2 são apresentados os critérios dessa rubrica.

A análise dos projetos finais entregues indica que os participantes adquiriram nível próximo ao máximo de proficiência previsto na rubrica nas seguintes categorias, apresentadas juntamente à média dos conceitos atribuídos: Paralelismo $(2,4)$, Raciocínio Lógico $(3,0)$, Sincronização $(2,6)$ e Controle de Fluxo $(2,6)$. A cobertura dos conceitos na escala proposta pela rubrica é apresentada na Figura 3. 
V Congresso Brasileiro de Informática na Educação (CBIE 2016)

Anais dos Workshops do V Congresso Brasileiro de Informática na Educação (CBIE 2016)

Tabela 2. Rubrica de avaliação da utilização de conceitos do Pensamento Computacional (MORENO-LEÓN; ROBLES, 2015)

\begin{tabular}{|c|c|c|c|}
\hline Conceito & Básico (1 ponto) & Intermediário ( 2 pontos $)$ & Avançado ( 3 pontos) \\
\hline $\begin{array}{l}\text { Abstração e decomposição } \\
\text { de problemas }\end{array}$ & $\begin{array}{l}\text { Mais de um script e mais } \\
\text { de um sprite }\end{array}$ & Definição de blocos & Uso de clones \\
\hline Paralelismo & $\begin{array}{l}\text { Dois scripts relacionados à } \\
\text { bandeira verde }\end{array}$ & $\begin{array}{l}\text { Dois scripts relacionados } \\
\text { ao pressionamento de } \\
\text { teclas ou clique no sprite } \\
\text { associados ao mesmo } \\
\text { sprite }\end{array}$ & $\begin{array}{l}\text { Dois scripts quando } \\
\text { receber mensagem, } \\
\text { criação de clones, dois } \\
\text { scripts quando } \% s>\text { s, } \\
\text { dois scripts quando o } \\
\text { pano de fundo mudar }\end{array}$ \\
\hline Raciocínio lógico & Uso do se & Uso do se...senão & Uso de operações lógicas \\
\hline Sincronização & Uso do espere & $\begin{array}{l}\text { Uso do envie mensagem a } \\
\text { todos, quando receber } \\
\text { mensagem, pare todos, }\end{array}$ & $\begin{array}{l}\text { Uso do espere até, quando } \\
o \text { pano de fundo mudar, } \\
\text { envie mensagem a todos e } \\
\text { espere }\end{array}$ \\
\hline Controle de fluxo & Sequência de blocos & Uso do repita e sempre & Uso do repita até que \\
\hline Interação com usuário & Uso da bandeira verde & 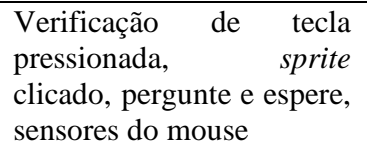 & $\begin{array}{l}\text { Estrutura quando \%s > } \\
\% s \text {, vídeo, audio }\end{array}$ \\
\hline Representação de dados & $\begin{array}{l}\text { Modificadores de } \\
\text { propriedades dos sprites }\end{array}$ & Operações em variáveis & Operações em listas \\
\hline
\end{tabular}

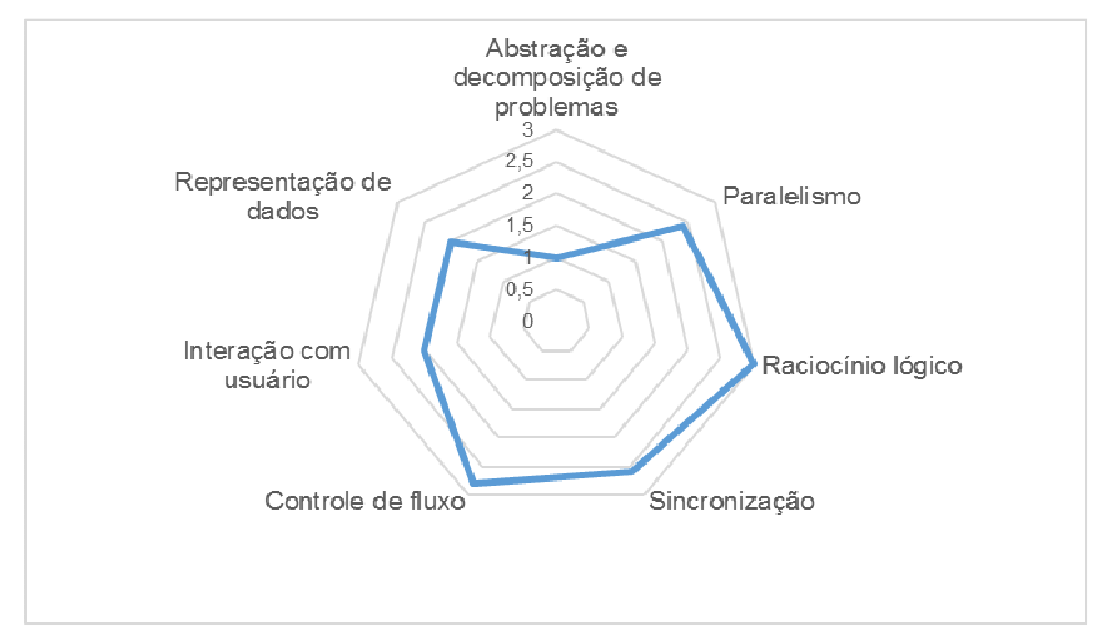

\section{Figura 3. Cobertura dos conceitos do Pensamento Computacional nos projetos finais desenvolvidos pelos participantes}

Esse é um resultado esperado pois a estrutura da oficina permitia o contato do aluno, em nível crescente de complexidade, com tópicos das categorias mencionadas. $\mathrm{Na}$ Tabela 3 é apresentado um mapeamento dos critérios da rubrica com os conteúdos da oficina, havendo uma menção se ao menos um dos critérios mencionados na rubrica está presente em algum dos módulos da oficina. É possível observar que, das quatro categorias da rubrica nas quais os participantes demonstraram uso proficiente dos conceitos, três são cobertas nas atividades da oficina até o nível avançado (Paralelismo, Raciocínio Lógico e Controle de Fluxo). Assim, em uma análise global, é possível inferir que os participantes, ao construírem uma aplicação a partir do zero, foram 
V Congresso Brasileiro de Informática na Educação (CBIE 2016)

Anais dos Workshops do V Congresso Brasileiro de Informática na Educação (CBIE 2016)

capazes de mobilizar conteúdos e competências muito próximos àqueles desenvolvidos ao longo da oficina. Cabe ainda ressaltar que, dos cinco participantes que desenvolveram o projeto final, quatro não tinham experiência anterior em programação de computadores.

Tabela 3. Mapeamento entre os critérios da rubrica e os conteúdos do curso.

\begin{tabular}{|l|c|c|c|}
\hline Conceito & Básico & Intermediário & Avançado \\
\hline Abstração e decomposição de problemas & $\mathrm{X}$ & $\mathrm{X}$ & \\
\hline Paralelismo & $\mathrm{X}$ & $\mathrm{X}$ & $\mathrm{X}$ \\
\hline Raciocínio lógico & $\mathrm{X}$ & $\mathrm{X}$ & $\mathrm{X}$ \\
\hline Sincronização & & $\mathrm{X}$ & \\
\hline Controle de fluxo & $\mathrm{X}$ & $\mathrm{X}$ & $\mathrm{X}$ \\
\hline Interação com usuário & $\mathrm{X}$ & $\mathrm{X}$ & \\
\hline Representação de dados & $\mathrm{X}$ & $\mathrm{X}$ & \\
\hline
\end{tabular}

Dentre os projetos finais desenvolvidos pelos alunos tivemos jogos do tipo quiz para treino de operações de soma, um puzzle para identificação de padrões em um painel de nove botões e um jogo de ação para identificação de números primos (Figura 4).

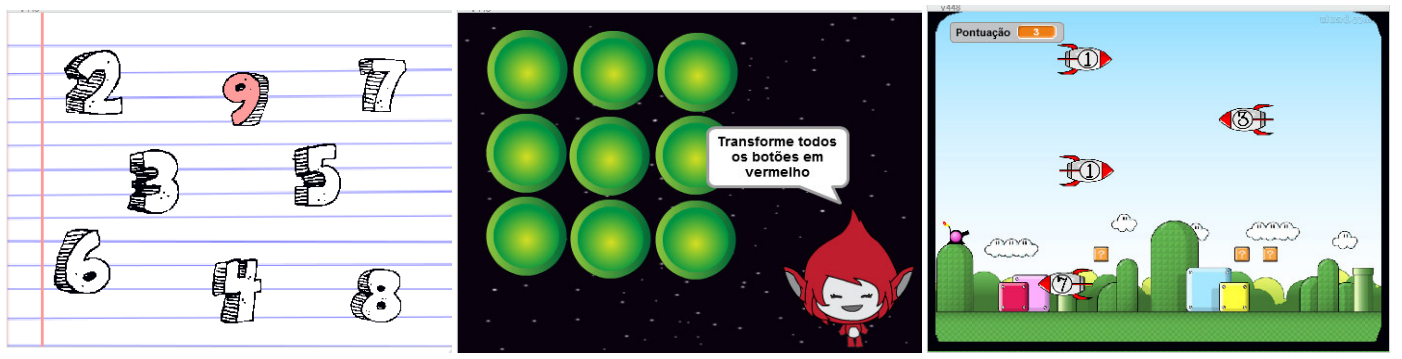

Figura 4. Exemplos de projetos finais desenvolvidos pelos alunos

\section{Conclusão}

A relevância do desenvolvimento do Pensamento Computacional na educação básica e o potencial de desenvolvimento conjunto com outras disciplinas vem sendo discutido ao longo dos anos. No entanto, a adequada formação de professores para trabalhar tais conceitos ainda se constitui como um desafio. Dessa forma, neste artigo é apresentada a estrutura e o oferecimento preliminar de um curso online para o desenvolvimento do Pensamento Computacional em professores de Matemática e estudantes de licenciatura em Matemática por meio do desenvolvimento de jogos digitais com a plataforma Scratch.

Os resultados preliminares indicam que o envolvimento dos participantes foi maior em atividades do curso que envolviam diretamente a construção de jogos, o que é um indício do aspecto motivador da temática escolhida. Ainda, os concluintes do curso demostraram por intermédio dos projetos finais um domínio intermediário ou avançado de aspectos do Pensamento Computacional relacionados à programação de computadores. Dessa forma, se tornaram autores de suas próprias atividades didáticas baseadas em jogos incluindo conceitos matemáticos.

Uma limitação do trabalho está associada às limitações de abrangência da rubrica utilizada para avaliação dos artefatos desenvolvidos; por outro lado, a análise isolada dos artefatos produzidos pode não ser capaz de revelar as práticas e concepções dos participantes (BRENNAN; RESNICK, 2012). Por essas razões, em trabalhos 
futuros pretende-se realizar um levantamento mais aprofundado do perfil dos participantes, bem como incorporar a coleta de dados por meio de entrevistas e questionários antes e após a participação na oficina. Ainda, pretende-se analisar possíveis razões para a evasão dos participantes. Dessa forma, espera-se identificar as decisões de design tomadas pelos participantes ao projetar seus próprios artefatos interativos e sua utilização dos novos conceitos aprendidos.

\section{Referências}

BARCELOS, T. et al. Relações entre o Pensamento Computacional e a Matemática: uma Revisão Sistemática da Literatura. In: IV CONGRESSO BRASILEIRO DE INFORMÁTICA NA EDUCAÇÃO E X CONFERÊNCIA LATINO-AMERICANA DE OBJETOS E TECNOLOGIAS DE APRENDIZAGEM. Anais dos Workshops do Congresso Brasileiro de Informática na Educação. Maceió: Sociedade Brasileira de Computação, 2015.

BARCELOS, T. S.; SILVEIRA, I. F. Computational Thinking and Mathematics: possible relationships revealed by an analysis of national curriculum guidelines. In: WANG, V. C. X. (Org.). Encyclopedia of Education and Technology in a Changing Society. $1^{\text {a }}$. ed. Hershey: IGI Global, 2014.

BLUM, L.; CORTINA, T. J. CS4HS: an outreach program for high school CS teachers. 2007, Covington, Kentucky, USA. Proceedings of the 38th SIGCSE technical symposium on Computer science education. Covington, Kentucky, USA: ACM, 2007. p. 19-23.

BRENNAN, K.; RESNICK, M. New frameworks for studying and assessing the development of computational thinking. In: AERA 2012, 2012, Vancouver. Proceedings of the 2012 annual meeting of the American Educational Research Association. Vancouver: American Educational Research Association, 2012.

CELLAN-JONES, R. A computing revolution in schools, 2014. Disponível em: <http://www.bbc.com/news/technology-29010511>. Acesso em: 29 abr. 2016.

CURZON, P. et al. Introducing teachers to computational thinking using unplugged storytelling. 2014, Berlin, Germany. Proceedings of the 9th Workshop in Primary and Secondary Computing Education. Berlin, Germany: ACM, 2014. p. 89-92.

FARIAS, A. B.; ANDRADE, W. L.; ALENCAR, R. A. Pensamento Computacional em Sala de Aula: Desafios, Possibilidades e a Formação Docente. In: IV CONGRESSO BRASILEIRO DE INFORMÁTICA NA EDUCAÇÃO E X CONFERÊNCIA LATINO-AMERICANA DE OBJETOS E TECNOLOGIAS DE APRENDIZAGEM. Anais dos Workshops do Congresso Brasileiro de Informática na Educação. Maceió: Sociedade Brasileira de Computação, 2015.

HOWLAND, K.; GOOD, J.; BOULAY, B. DU. Narrative support for young game designers' writing. Proceedings of the 14th International Conference on Interaction Design and Children. Boston, Massachusetts: ACM, 2015. p. 178-187.

KE, F. An implementation of design-based learning through creating educational computer games: A case study on mathematics learning during design and computing. Computers \& Education, v. 73, p. 26-39, abr. 2014. 
MORENO-LEÓN, J.; ROBLES, G. Dr. Scratch: a web tool to automatically evaluate Scratch projects. 2015, London. Proceedings of the 10th Workshop in Primary and Secondary Computing Education. London: ACM, 2015. p. 132-133.

MORREALE, P.; JOINER, D. Changing perceptions of computer science and computational thinking among high school teachers. J. Comput. Sci. Coll., v. 26, n. 6, p. 71-77, 2011.

MOR, Y.; NOSS, R. Programming as mathematical narrative. International Journal of Continuing Engineering Education and Life-long Learning, v. 18, n. 2, p. 214-233, 2008.

MUÑOZ, R. et al. Diseño e Implementación de un Taller de Programación de Juegos Digitales con Scratch como Apoyo a Fundamentos de Programación. In: IV CONGRESSO BRASILEIRO DE INFORMÁTICA NA EDUCAÇÃO E $X$ CONFERÊNCIA LATINO-AMERICANA DE OBJETOS E TECNOLOGIAS DE APRENDIZAGEM. Anais dos Workshops do Congresso Brasileiro de Informática na Educação. Maceió: Sociedade Brasileira de Computação, 2015.

PEPPLER, K.; KAFAI, Y. Gaming Fluencies: Pathways into Participatory Culture in a Community Design Studio. International Journal of Learning and Media, v. 1, n. 4, p. $45-58,1$ nov. 2009.

QIN, H. Teaching computational thinking through bioinformatics to biology students. SIGCSE Bulletin, v. 41, n. 1, p. 188-191, mar. 2009.

SERVIÇO BRASILEIRO DE APOIO ÀS MICRO E PEQUENAS EMPRESAS. O panorama e a evolução do mercado de "games" no Brasil. Disponível em: <http://www.bibliotecas.sebrae.com.br/chronus/ARQUIVOS_CHRONUS/bds/bds.nsf/9 d1a01803afb08188249685444987b01/\$File/4287.pdf>. Acesso em: 20 ago. 2016.

SHADISH, W. R.; COOK, T. D.; CAMPBELL, D. T. Experimental and quasiexperimental design for generalized causal inference. Boston: Houghton Mifflin, 2002.

THE CSTA STANDARDS TASK FORCE. CSTA K-12 Computer Science Standards. New York: ACM Computer Science Teachers Association, 2011. Disponível em: <http://csta.acm.org/Curriculum/sub/CurrFiles/CSTA_K-12_CSS.pdf〉. Acesso em: 3 fev. 2012.

YADAV, A. et al. Computational Thinking in Elementary and Secondary Teacher Education. Trans. Comput. Educ., v. 14, n. 1, p. 5:1-5:16, mar. 2014. 